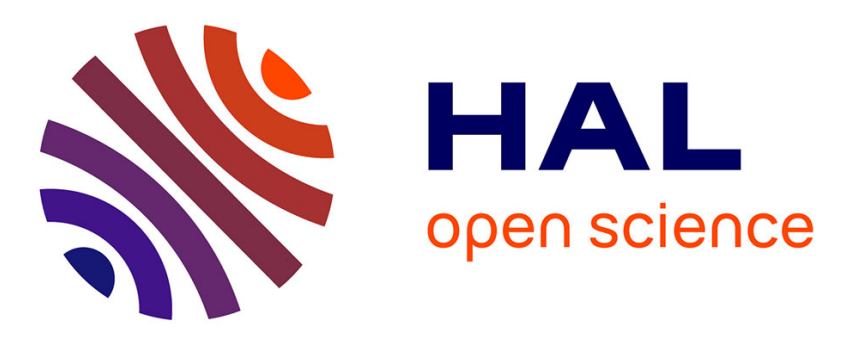

\title{
Multimedia Content Delivery for Remote Patient Monitoring using Cognitive Radio Networks
}

Dramane Ouattara, Mohamed Aymen Chalouf, Omessaad Hamdi, Francine

Krief

\section{- To cite this version:}

Dramane Ouattara, Mohamed Aymen Chalouf, Omessaad Hamdi, Francine Krief. Multimedia Content Delivery for Remote Patient Monitoring using Cognitive Radio Networks. WTS 2014, the 13th annual Wireless Telecommunications Symposium, Apr 2014, Washington, DC., United States. 8 p. hal00998855

\section{HAL Id: hal-00998855 \\ https://hal.science/hal-00998855}

Submitted on 2 Jun 2014

HAL is a multi-disciplinary open access archive for the deposit and dissemination of scientific research documents, whether they are published or not. The documents may come from teaching and research institutions in France or abroad, or from public or private research centers.
L'archive ouverte pluridisciplinaire HAL, est destinée au dépôt et à la diffusion de documents scientifiques de niveau recherche, publiés ou non, émanant des établissements d'enseignement et de recherche français ou étrangers, des laboratoires publics ou privés. 


\title{
Multimedia Content Delivery for Remote Patient Monitoring using Cognitive Radio Networks
}

\author{
Dramane Ouattara*, Mohamed Aymen Chalouf $f^{\dagger}$ Omessaad Hamdi* and Francine Krief* ${ }^{*}$ \\ * Univ. Bordeaux, LaBRI, Talence, France. \\ Email: dramane.ouattara@labri.fr, ohamdi@labri.fr, francine.krief@labri.fr. \\ $\dagger$ Univ. Rennes 1, IRISA, Lannion, France Email: mohamed-aymen.chalouf@irisa.fr \\ $\ddagger$ IPB, LaBRI, Talence, France.
}

\begin{abstract}
Medical multimedia data transmission for remote diagnosis and emergency incident reports will significantly increase in the coming years. Thus, broadband network technologies with ability to be adaptable to user's context and application content must be considered. In this paper, we propose an on-the-fly resource reservation protocol for medical multimedia content delivery in cognitive radio networks. Our approach is based on an appropriate channels switching, that meets the need of end-to-end video quality and throughput requirements for better clinicians analysis. When a channel switching must be performed, an appropriate channel is reserved. This process will also rely on a signalling protocol which allows transmitting traffic characteristics and required network resources to all relay nodes for a best decision-making.
\end{abstract}

\section{INTRODUCTION}

Assistive technologies, remote diagnosis and care, elderly and chronic diseases patients monitoring are services that contribute to medical multimedia content delivery increasing. Hence, medical multimedia data delivery over wireless networks is expected to grow drastically. It is also important to note that data transmission success depends on bandwidth availability in wireless networks and videocompression effectiveness. Therefore, the most important components in designing wireless medical multimedia data transmission framework are the effective medical data compression technology and the appropriate wireless infrastructure. Medical content transmission over networks is constrained by the communication channels capacity. In this regard, efficient data compression systems can be build based on the existing encoding standards such as H.264/AVC [1]. Also, given the scarcity of spectrum resources and its inefficient use, the cognitive radio networks are well suited for medical and emergency services. Cognitive Radio Networks (CRN) [2] have recently emerged as a new wireless technology that deals with the shortage of radio resources and the inefficiency in spectrum usage. In most common configurations, a Cognitive Radio (CR) user exploits the spectrum bands transparently to legacy users also called Primary Users (PU). Using its flexibility, the $\mathrm{CR}$ device explores different technologies and standards to identify free frequency bands for opportunistically en- suring its transmissions. The cognitive radio have recently become the forefront of wireless research, and promises to reduce connection cost and offers higher throughput. This technology could facilitate medical multimedia transmissions with more flexibility. However, multimedia delivery over cognitive radio networks involves challenges such as the spectrum sensing, the latency, the error control and the primary user protection. In this paper, we propose a best effort on-the-fly resource reservation, carrying the data flow end-to-end with a new scheduling plan that reduce latency and improve throughput. The remainder of this paper is structured as follows: in section II, we make an overview on the related work. Then, we give an analysis of the delay in Cognitive Radio Networks in section III. We present the medical multimedia transmission context in section IV. In section V, we propose a function that ensures communication reliability in resource sharing constraints for clinical multimedia data transmissions. Section VI shows and analyses simulation results. Finally, section VII concludes this work and highlights some interesting perspectives.

\section{RELATED WORK}

Today, studies on delivering medical multimedia content are focusing on compression techniques, encoding methods, video resolution methods to meet the needs of adapting to the network parameters[3][4]. Adaptation to wireless parameters means in that case, diagnosticallyrelevant selection of the source encoding parameters and the error control methods. E-health multimedia systems have been primarily based on $3 \mathrm{G}$ wireless networks [5]. However, given the limited upload data rates (up to 384 Kbps), as shown in [6], the associated source encoding parameters were bounded to different medical video resolution sizes/types. Researchers are now more focusing on $3.5 \mathrm{G}$ and $4 \mathrm{G}$ wireless technology for delivering high resolution and video rate with low delay and low packetloss rate [7]. This evolution from $3 \mathrm{G}$ to $4 \mathrm{G}$ offers broadband multimedia transmissions but does not solve the spectrum scarcity issues and its inefficient usage. Also, it is obvious that $4 \mathrm{G}$ technology is not available everywhere and at any-time. In this context, researchers are inter- 
ested in the use of cognitive radio for medical multimedia transmissions. In [8], video encoding rate, power control, relay selection and channel allocation are jointly considered for transmission over cooperative cognitive radio networks. The problem is formulated as a mixed-integer non-linear problem and solved by an algorithm based on a combination of the branch and bound framework and convex relaxation techniques. This contribution should take into account delay that is a very important constraint in real-time medical multimedia flow transmission. In [9], an algorithm is proposed to evaluate the expected delay of competing flows in single-hop and two-hops networks considering the time-varying spectrum condition and occupancy, traffic characteristics, and the condition of queues at intermediate nodes. Simulation results show that the proposed algorithm reduces the packet loss rate and improves the average Peak Signal-to-Noise Ratio (PSNR) of the received video streams. The work presented in [10] considers the multi-user bit-rate and latency control of scalable video content in a cognitive radio multimedia network. The proposed model is based on CRN where multiple secondary users attempt to access a spectrum hole according to a predefined Time Division Multiple Access (TDMA) rule based on primary user activities, channel quality and transmission delay of each user. Scalable video rate and distortion models have been used in formulating the problem as a switching control dynamic Markovian game. Video sources and channel behaviour have been modelled as independent Markov processes. This work shows that the proposed switching control game formulation results in an improvement in video quality over a myopic rate allocation scheme in video PSNR. To the above mentioned transmission optimisation efforts, are added scheduling mechanisms used for traffic prioritization. In [11], a priority virtual queue model is adopted for wireless CR users to select channel and maximize video quality. Simulation results show that the proposed channel selection and access solution based on priority scheduling outperforms the conventional dynamic channel selection scheme by $2 \mathrm{~dB}$ (PSNR). Most of the research papers consider traffic prioritization as the best strategy to improve multimedia data transmissions quality. However, the classification of traffic has significant limitations in terms of waiting time and therefore increases the end-toend latency as we will demonstrate in section III. Our proposal has the advantage of improving the scheduling process under data-flow prioritisation with an on-the-fly reservation method, which ensures continuity of service, while reducing waiting delays. This proposed improvement in the channel sharing process seems interesting for delivering high bit-rate multimedia-data with low delay and low packet-loss that could help to approach the experience of in-hospital examinations.

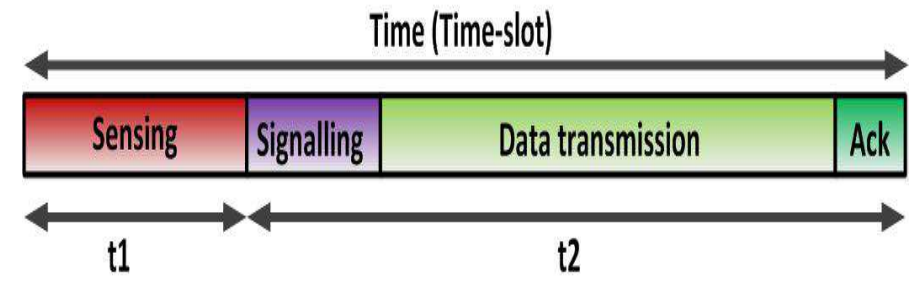

Fig. 1. Cognitive radio medium access method

\section{Cognitive RADIO DELAY ANALYSIS}

\section{A. Theoretical analysis}

In CRN, we have two types of users: Primary Users (PU) who have the license to use bands and Secondary Users (SUs) also called cognitive radio users that explore opportunistically the free frequency bands. The sensing module of cognitive radio allows the detection of free bands and the sharing module allows to control their access. Figure 1 depicts the medium access process in cognitive radio networks. Sensing phase with duration $t 1$, that occurs before any transmission seems very important. More this phase $(t 1)$ is longer, more it has better detection results of free spectrum and better control of interferences. Signalling phase corresponds to the period of information exchange, necessary for the communication session initiation. It allows to reach an agreement on the criteria of communication (e.g. frequency band), taking into account applications constraints. Also, a significant lapse of time should be reserved for data transmission after signalling and a time for acknowledgement as shown in figure 1. A "Time-slot" $(t 1+t 2)$ is the total time given to a cognitive radio user for transmitting a data packet. This time-slot is obviously longer than in standards networks where medium access process corresponds to the time $t 2$ of figure 1. As shown in Figure 1, there is a significant latency and delay linked to the sensing that could affect the quality of real-time medical video transmission in cognitive radio networks.

\section{B. Latency estimation by simulations}

Sensing is obviously inevitable and the quality of free bands detection is related to the sensing duration in CRN. The time allocated to the sensing activity appears incompressible. So, to reduce latency and delay, it is more appropriate to target signalling and transmission periods. We experienced the waiting time and the importance of the delay through simulations. The results of our simulations (Figure 2), show the behaviour of scheduling algorithm that assigns the highest priority to medical multimedia traffic according to the time-slot principle. In a simulation-function, we created 10 data packet queues with a priority assigned to each queue classified from 0 (highest priority) to 9 (lowest priority). Here, the highest priority is associated to medical multimedia contents and the duration of a time-slot is equal to 10 seconds. In Figure 
2 , the queue in running is coloured in red while blue queues are waiting.

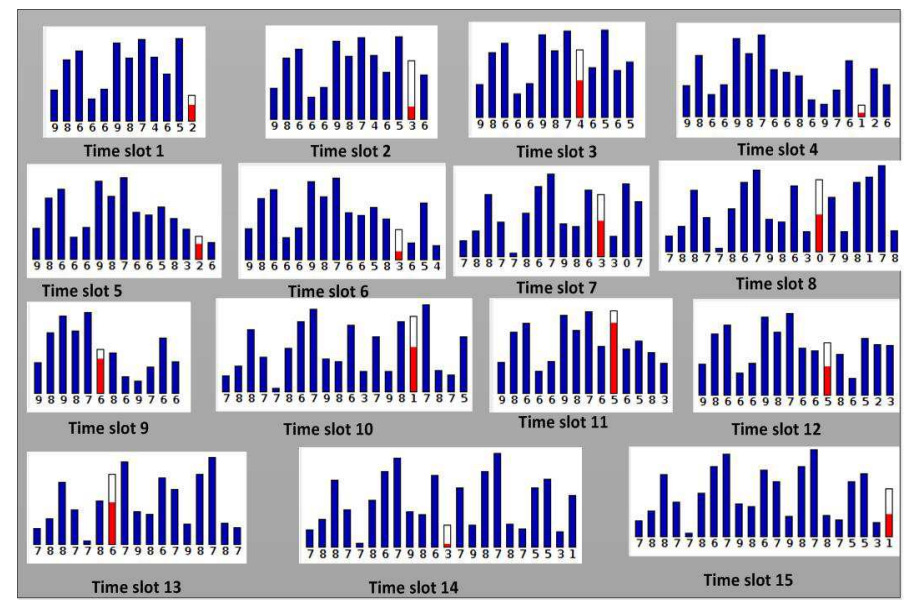

Fig. 2. Simulation results

The execution of the simulation function shows that the waiting time depends on the arrival of a highest priority queue during the execution of a time-slot allocated to a lower priority queue as viewed in Figure 2. The most illustrative example, concerns the time-slot 7 where the queue with priority 3 runs while the queue with priority 0 ( the highest priority) is waiting. It is only at the end of the time-slot 7 that queue with priority 0 starts its transmission that corresponds to time-slot 8 . This confirms the delay already mentioned in the theoretical analysis that could generate a high latency and have an impact on the quality of medical multimedia end-to-end transmission. Knowing that the time-slot is an atomic operation, there is a real latency to consider and to overcome in cognitive radio networks dedicated to emergency medical traffics.

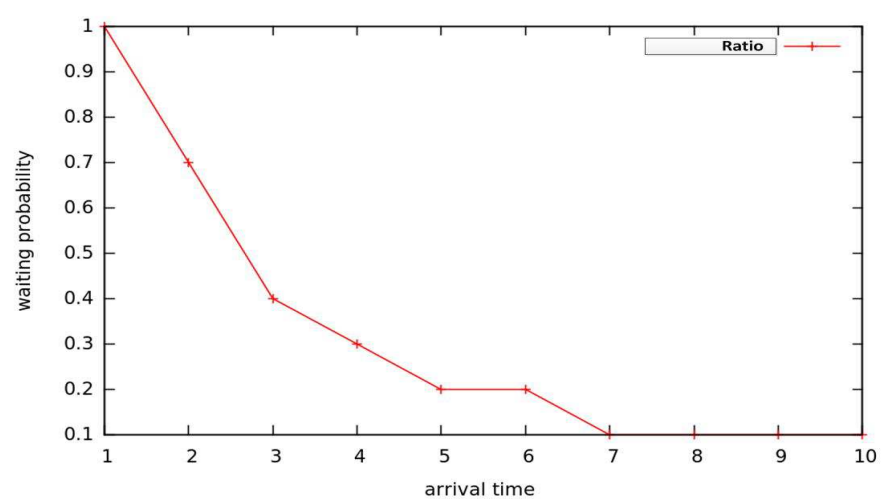

Fig. 3. Ratio of waiting time

Figure 3 represents the results that express the time lost in waiting for the end of execution of a time-slot in progress. This confirms the significant delay that affects the transmission quality in systems with traffic prioritization. It should be noted that more the priority traffic is reported at the beginning of the execution of a timeslot, more the waiting time is higher, even if this time is relative to the total duration of a time-slot. It is also clear from these results that for different traffics with the same priority, the waiting time becomes inevitably more important for pending queues and may be greater than the duration of a time-slot. This requires improvements in the context of medical multimedia data transmissions, that are more sensitive to delay and latency.

\section{Medical multimedia-DAta transmission CONTEXT}

Early diagnosis of patients at remote locations is expected to reduce patients risks to more general diseases (e.g. cardiovascular diseases). Basically, the idea is to communicate vital signs such as electrocardiogram, heart rate, blood pression and medical multimedia data to an emergency physician. Receiving medical data in real-time allows caregivers to further assist the diagnosis and to prepare the patient's admission to the hospital. From Figure 4, the key concept is to communicate the patient's clinical video or multimedia data to the hospital premises, for remote diagnosis and assistance using monitoring equipments residing in the ambulance or at home. This scenario highlights the technological challenges associated to wireless communications and therefore with cognitive radio networks for adequate diagnostic quality. Indeed, the varying state of the wireless channels and bandwidth contributes to multimedia transmission quality degradation when transmitting from the patient's home or from the ambulance to the hospital. Thus, permanent connectivity and reliability as studied in [12], are the major challenges. So, maintaining connection with a high quality of service for medical multimedia content is the main purpose of this work.

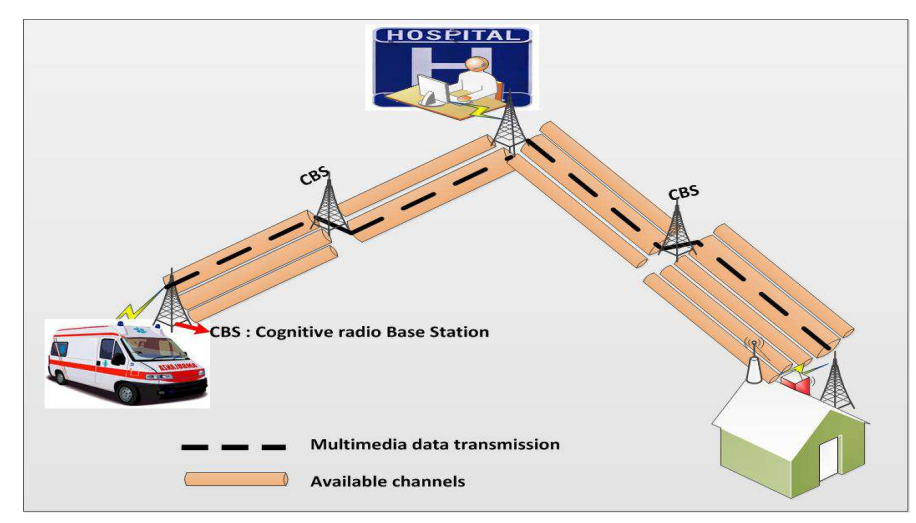

Fig. 4. The proposed transmission context

In this scenario, we investigate the establishment of the communication and ensure its maintenance for reliable end-to-end multimedia transmissions. The main idea is that, Cognitive Base Station (CBS) exchange information through a signalling protocol for determining the 
path composed of free frequency-band useful for medical multimedia flows end-to-end delivery. The connexion is initiated through a message that contains traffic characteristics and needed network resources. This message is sent to all hosts or relay nodes (CBS) to request the required QoS for medical multimedia content transmissions. In this process, each node makes its decision independently, based on shared/received information and locally resources availability. It is important to note that routing protocols [13][14] in cognitive radio networks allows selecting the route that satisfies both primary users avoidance and secondary users end-to-end communications achievement. Indeed, with routing protocols, the main information exchanged is channel availability which is obtained from spectrum sensing mechanism or spectrum occupancy database. The signalling protocol considered is a Medium Access Control layer protocol that provides channel sharing information and allows taking into account the applications QoS constraints. It allows exchanging information about content type, flow priority and recommended QoS to allow each node constituting the previously established path (with the routing protocol), to make resource sharing decisions autonomously as more detailed in [15]. Thus, our proposal relies on the ability to find the adequate network resources and the adoption of strategies to achieve full transmissions despite the channel changing constraints (e.g. frequency hopping during a transmission).

\section{Maintaining tRAnSmissions in CHANnel SHARING CONSTRAINTS}

At each time-slot $t$, the base station selects a set of channels to sense and a set of channels to access. Each $\mathrm{CR}$ user can access all available channels with the channel bonding or channel aggregation techniques [16]. Spectrum sharing is based on the list of free-channels contained in the database (Figure 5). To maintain the transmission and its QoS despite the arrival of a Primary User, we propose to add a sub-process (P2) to the existing cognitive radio sharing-scheduling module $(P 1)$. The sub-module $P 2$ is activated when a medical multimedia stream is detected through signalling process. The operating mechanism of $P 1$ is more detailed in [11]. Regarding the operation of $P 2$, a first resource is allocated for the establishment of the communication (Figure 5). Then, one or more channels are reserved on-the-fly, when needed, to compensate the possible unavailability of the channel in use. Unavailability could be related to the Primary Users activities or to inappropriate band quality for medical multimedia content delivery. At least two free frequency bands will be necessary for this resource sharing algorithm to be runnable.

An adapted resource sharing process allows reducing the latency induced by the time-slots as explained in section III. The on-the-fly resource reservation that we propose allows avoiding the monopolization of a frequency resource that will not be used. To achieve this goal, a function for

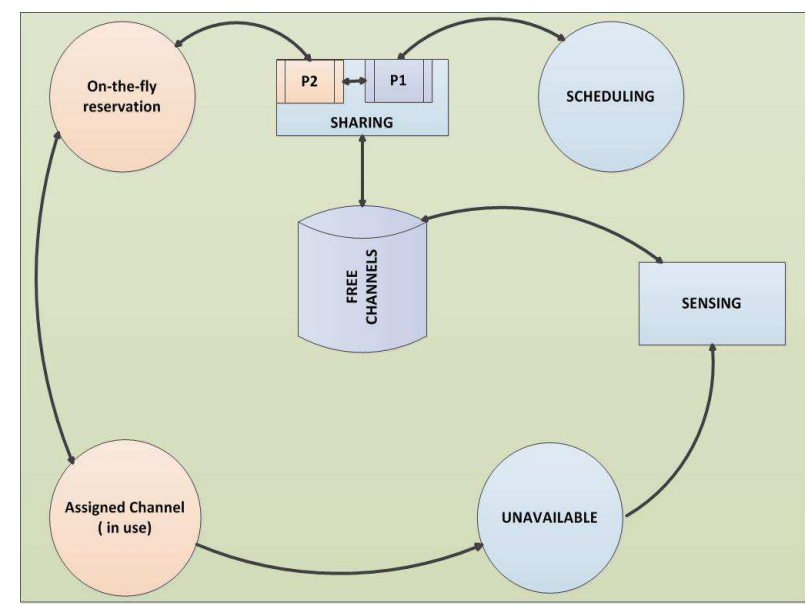

Fig. 5. Channel sharing and scheduling process

control and monitoring of the transmission parameters is necessary. To make efficient use of the spectrum resource by avoiding reservation without switching on the given band, we propose the Model Predictive Control(MPC)[17] as the basis of decision-making (reserve or do not reserve). The principle of this on-the-fly MPC-based reservations is composed of a set of rules:

- Evaluation of transmission parameters: (throughput, packet loss rate, delay), whose values can be obtained through real-time QoS measurement/statistic tools such as the QoS Metrology (QoSMet) ${ }^{1}$ and channel availability information given by the sensing module.

- Decision: At each time-slot $t$, the $M P C$ module calculates and predicts transmission conditions (variation of parameters) characterising the time-slot $t+1$.

- If transmission conditions are good for the time-slot $t+1$ then, no channel is reserved. This means that for the time-slot $t+1$, there is a high probability of maintaining the quality of transmission to an acceptable level for the medical multimedia data delivery.

- Otherwise, a broadcast message through the signalling protocol is sent, indicating that a channel $\theta$ is reserved for the time-slot $t+1$.

\section{A. Channel reservation modelled with the $M P C$}

Model Predictive Control is a technique that adapts to the dynamics of a process by its real-time controller to predict the future behaviour of this process. Thus, the unstable nature of the transmission parameters and the cognitive radio channel will be controlled to optimize the use of resources for the future time-slots while taking into account the medical multimedia data transmission quality. In our proposal, the $M P C$ will compute the optimal decision between staying on the current channel without reserving or moving to another frequency band with prior reservation, taking into account constraints and

\footnotetext{
${ }^{1}$ http://michaut.valerie.free.fr/qosmet/
} 
disturbances. The main advantage of $M P C$ compared to other statistical prediction models based on the history data, is its ability to achieve optimized predictions by implementing only the current time-slot with less calculation [18].

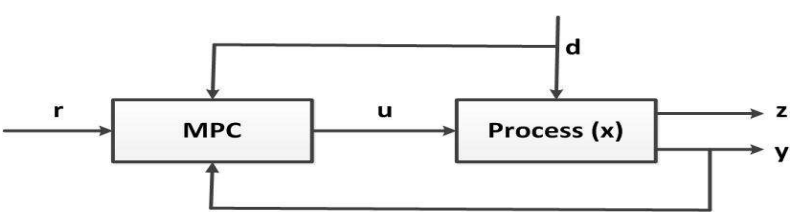

Fig. 6. The considered MPC-model

1) Description of the $M P C$ basic idea: Figure 6 illustrates the basic idea for the $M P C$ where $r$ is the set-points describing the reference values of transmission parameters. From these reference variables, the controller manipulates the inputs $u$, for achieving predictions through outputs $z$. The output values $z$ are then compared with measured values $y$, for ensuring the relevance of outputs while controlling their compliance with the set-points as possible. Variable $\mathrm{d}$ is the disturbance linked to a Primary User signal, and any other noise that could degrade transmissions quality. The principle is to monitor in real time the evolution (at time-slot $t+1$ ) of the parameters $(z)$ of medical data and make a decision on the basis of established criteria and constraints.

2) Modelling the MPC for channel reservation: The $M P C$ is based on a transfer function $\mathrm{H}(\mathrm{x})$ that expresses the relation between each input $u$ and output $z$ variables as follow: $H(x)=\frac{Z(x)}{U(x)}$

We then deduce the following optimization function given below:

$$
\phi=\frac{1}{2} \sum_{i=1}^{N} w_{z_{i}}\left\|\left(z_{i}-r_{i}\right)\right\|^{2}+\frac{1}{2} \sum_{i=1}^{N-1} w_{u_{i}}\left\|\Delta u_{i}\right\|^{2}
$$

With $\phi$, we can predict the future sequence, when there exist $\mathrm{n}$ transmission parameters to control (output variables) and $m$ other transmission parameters to manipulate as inputs. This prediction is detailed by the function:

$$
\begin{aligned}
\Phi= & \sum_{i=1}^{n}\left(\sum_{j=1}^{j=N z_{i}} w_{z_{i}} \|\left(z_{i}(t+j)-r_{i}(t+j) \|^{2}\right)+\right. \\
& \sum_{i=1}^{m}\left(\sum_{j=1}^{j=N u_{i}} w_{u_{i}} \|\left(z_{i}\left(\Delta u_{i}(t+j-1)\right) \|^{2}\right)\right.
\end{aligned}
$$

Coefficients $w_{z_{i}}$ and $w_{u_{i}}$ are weights that can give more importance to a given parameter. To take into account the constraints of the transmission environment, MPC offers the possibility to assign different types of constraints (output and input) on the network parameters. These constraints can be summarized as follow:

Prediction $x_{i+1}=A x_{i}+B u_{i}+E d_{i}$ for $i=0,1,2, \ldots ., N-1$
Where:

$z_{i}=C x_{i}$, for $i=0,1,2, \ldots \ldots, N$

$u_{\text {min }} \leq u_{i} \leq u_{\max }$ for $i=0,1,2, \ldots \ldots, N-1$

$\Delta u_{\min } \leq \Delta u_{i} \leq \Delta u_{\max }$ for $i=0,1,2, \ldots \ldots, N-1$

$z_{\text {min }} \leq z_{i} \leq z_{\max }$ for $i=0,1,2, \ldots \ldots, N$

We can note that the prediction at time-slot $t_{0}$ is not considered, and that gives the future values with the following constraints:

$$
\begin{aligned}
& z_{\min } \leq z_{i} \leq z_{\max } \quad \Rightarrow \\
& {\left[\begin{array}{c}
z_{\min } \\
z_{\min } \\
\cdot \\
\cdot \\
z_{\min }
\end{array}\right] \leq\left[\begin{array}{c}
z_{1} \\
z_{2} \\
\cdot \\
\cdot \\
z_{N}
\end{array}\right] \leq\left[\begin{array}{c}
z_{\max } \\
z_{\max } \\
\cdot \\
\cdot \\
z_{\max }
\end{array}\right]}
\end{aligned}
$$

A cognitive radio node could continue to occupy a given channel-band for its transmission of medical data only if these constraints are respected.

\section{B. Implementation of the channel reservation with $M P C$}

We used Matlab for our reservation approach implementation. Simulations are performed according to the relevance of parameters, in real-time multimedia data transfer context. These transmission variables are: delay, throughput, bandwidth, transmit power and channel threshold. For each parameter, we defined the inherent constraints as detailed in Table I. The limits in Table I, are

\begin{tabular}{l|l} 
Parameters & Descriptions \\
\hline \hline$N$ & Prediction horizon size \\
\hline$u_{\min }$ & $\begin{array}{l}\text { Lower limit of each transmission parameter linked } \\
\text { to } \mathrm{u}\end{array}$ \\
\hline$u_{\max }$ & $\begin{array}{l}\text { Upper limit of each transmission parameter linked } \\
\text { to } \mathrm{u}\end{array}$ \\
\hline$\Delta u_{\min }$ & Rate of change of the parameter $u_{\min }$ \\
\hline$\Delta u_{\max }$ & Rate of change of the parameter $u_{\max }$ \\
\hline$z_{\min }$ & Lower limit of each predicted parameter \\
\hline$z_{\max }$ & Upper limit of each predicted parameter \\
\hline$x_{0}, u_{0}$ & Simulation starting points \\
\hline$w$ & $\begin{array}{l}\text { Weight matrices related to each parameter } \\
\text { importance }\end{array}$ \\
\hline
\end{tabular}

TABLE I

all constraints to be respected by the cognitive radio node to continue using the channel-band without triggering the reservation process. The reservation process is triggered on the fly only when these instructions and these constraints are violated. This overcomes the risk of reserving a channel without accessing to it in time-slot $t+1$. It also guarantees a better use of the available bands and offers an efficient sharing of the spectrum resources. The Matlab code below (Figure 7) shows how the control of variables (delay, packet loss, bandwidth) is implemented. The parameters chosen for the purpose of these simulation are based on values proposed in the accompanying guide ${ }^{2}$ for expression of

${ }^{2}$ www.datar.gouv.fr 
infrastructure needs high and very high speed to deploy telemedicine projects in France. Thus, the recommended delay for real-time telemedicine applications has to be less than 15 milliseconds for 100 bytes of data. The minimum throughput to ensure quality of service is $10 \mathrm{Mbps}$ and packet loss is fixed at 0.2 percent maximum for 100 bytes of data. Knowing that the multimedia transmission needs a large bandwidth, we set the following constraints in Matlab code as described in Figure 7. These constraints and conditions could be dynamic and change depending on the technology used and the radio environment.

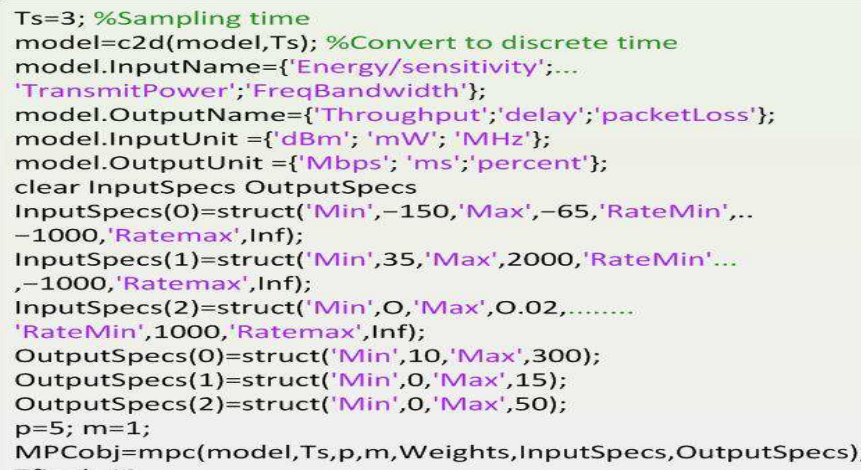

Fig. 7. Constraints set for simulation in Matlab

The code shown in Figure 7 includes the limits fixed for the variables (InputSpecs, OutputSpecs), and the constraints to be respected. In our simulations, we set a couple of values (RateMin, Ratemax) that determine the frequency of changes of each variable and another value (p) for the prediction horizon.

\section{Results AND ANALYSIS}

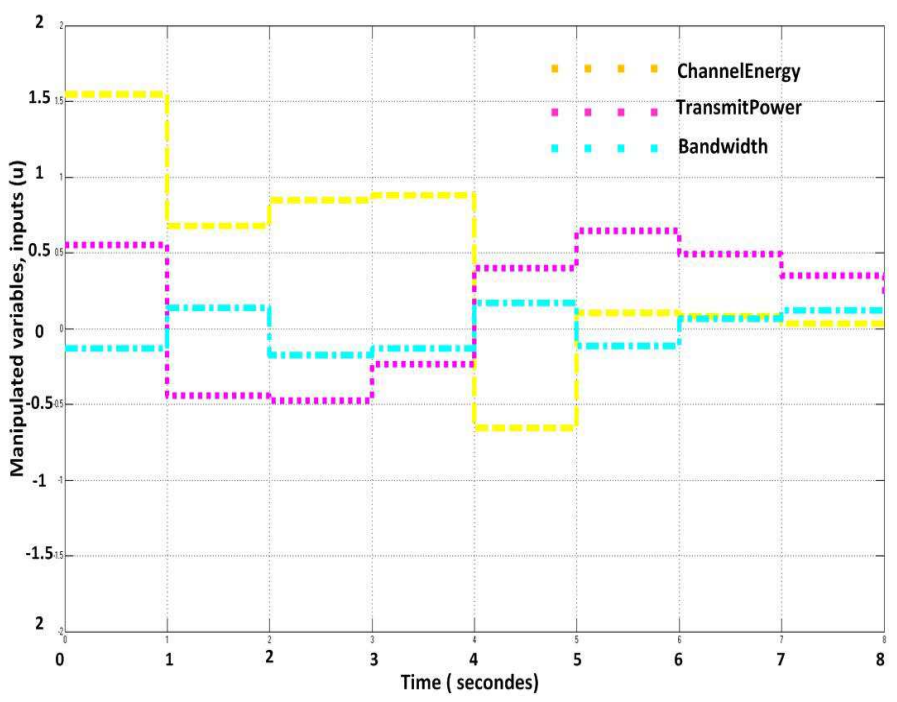

Fig. 8. Input variables variation

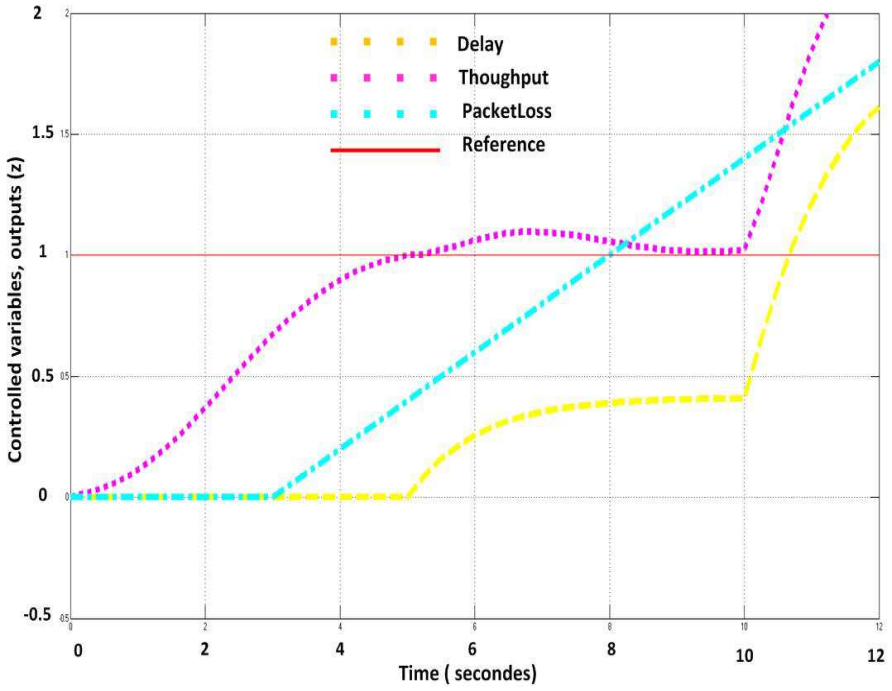

Fig. 9. Output variables variation without equilibrium

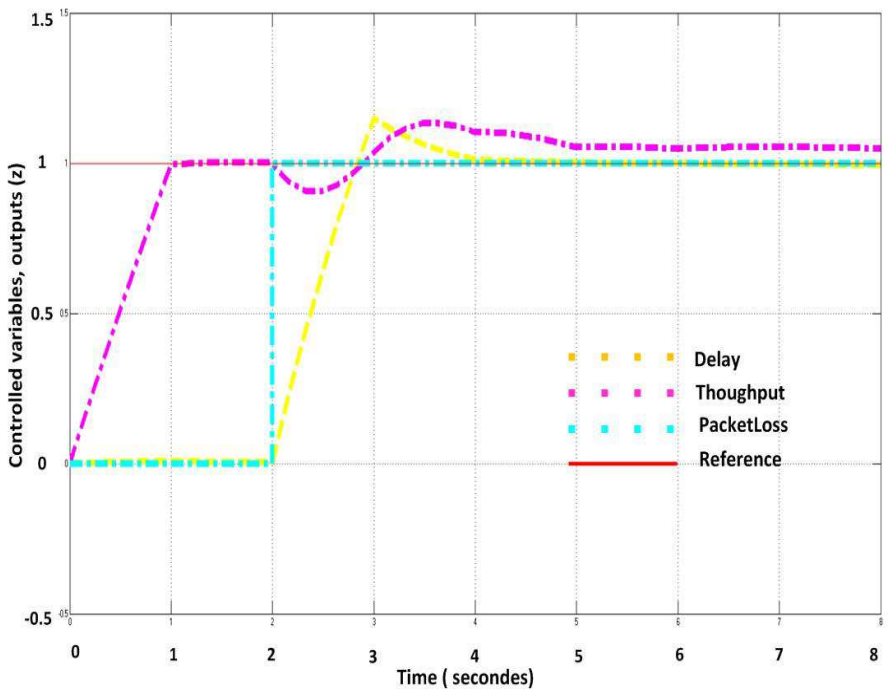

Fig. 10. Output variables variation with equilibrium

Before describing the obtained results, it should be noted that the $M P C$ toolbox functions (autosc, scal and wrtreg) allow us to arrange all data (input variables and output variables) in the same order of magnitude. The predictions and obtained results are based on these reestimated values. The objective remains minimizing a quadratic criterion based on prediction errors up to an order equal to the relative degree between the predicted outputs/inputs variables and the reference values often measured. Results show the vectors of predicted future errors over the horizon $p$ which result from all future manipulated variables. The main objective is to reach a stability of the system in time-varying manipulate variables (inputs, outputs). This stability reflects the fact that the desired QoS, with constraints on the output variables is achieved through dynamic adaptation of the input parameters (delay, packet loss, throughput). We 
tested different scenarios and for the first one, we set the constraints/limits on input and output variables. The constraints are fixed and the values of the input parameters are dynamically adapted to meet these constraints. For the second scenario, we set the constraints/limits only on the output variables and try to make an adjustment of input variables as described at Figure 8, to meet these constraints. In the last scenario, we set constraints and limits on the input variables and observe the variation of the outputs. We see through these simulations that the results of the second scenario and the last one are almost identical and the system has difficulties to stabilize or does not give satisfying results as shown in Figure 9. This means that the conditions to ensure an acceptable level of QoS are not met. Figure 10 shows that when the constraints are set on both the input and the output variables (first scenario), the system quickly stabilizes. This means that the input parameters dynamically selected, correspond to the constraints and their impact on output values is in accordance with the QoS requirements. The first scenario seems to be the correct formulation of the model for better control of the parameters, faster execution and more accurate results. Thus, the interest here is that, while the system converges and becomes stable then it is not necessary to make a resource reservation for the next timeslot. In case the system fails to stabilize (Figure 9) or it loses its stability during a time-slot, then a reservation resource for next time-slot becomes relevant. The great advantage from these results is to have shown that in our system with cognitive radio equipments, it become possible to fix constraints (intervals) on input variables and to allow the equipments keeping their adaptable character by choosing their transmission parameters based on the radio environment with respect to the output intervals/constraints.

\section{Conclusion}

In this paper, we proposed the use of cognitive radio as network infrastructure for the transmission of medical multimedia data. For communication's performances evaluation, we made a theoretical analysis of the delay, caused by resource sharing in CRN. Theoretical results are validated by simulations and show the limits of the resources sharing and their likely impact on the quality of medical multimedia transmissions. To improve performance and provide better quality of service, we proposed adding to the resource-sharing module, an on-the-fly reservation function for emergency traffic. This function is based on the MPC-module that allows to control the transmission variables and to predict for the next time-slot, if a resource reservation is needed or not. Our proposal offers better strategy for resource sharing in a context with different types of traffic. It also helps maintaining QoS and reduces waiting delay. More precisely, our proposal allows us to highlight the following main outlines:
- Transmission of medical multimedia content, is based on a distributed decision-making process.

- Each cognitive radio node performs the best effort as possible to choose the best input parameters and ensuring quality of outputs based on the local constraints (imposed by the MPC-module) and the local resource availability.

- With the control of output parameters and dynamic adjustment of input variables within the fixed constraints, it becomes possible to make the appropriate decision and to proceed with reservation when needed.

- The user is no longer forced to leave the channel with the detection of a primary user. It becomes possible to adapt the transmission parameters and continue sharing the channel.

Hence, our proposal allows ensuring permanent connectivity for medical multimedia data transmissions while decreasing latency, waiting delay and improving throughput. The future work will consists in implementing the proposed solution on our cognitive radio platform to confirm simulation results.

\section{ACKNOWLEDGMENT}

This work is supported by the LICoRNe project, funded in part by the National Agency for Research in France ANR (Agence Nationale de la Recherche).

\section{REFERENCES}

[1] Lopez, J.P.; Jimenez, D.; Cerezo, A.; Menendez, J.M.:Noreference algorithms for video quality assessment based on artifact evaluation in MPEG-2 and H.264 encoding standards: IFIP/IEEE International Symposium, 2013

[2] Mitola, J.; Maguire, G.Q., Jr.: Cognitive radio: making software radios more personal: Personal Communications IEEE, 1999.

[3] Jingfang Huang; Zhaoyang Zhang; Honggang Wang; Hong Liu : Video transmission over Cognitive Radio networks : GLOBECOM Workshops (GC Wkshps), 2011 IEEE, 2011

[4] Mingjing Ai; Dairui Cui; Shaopeng Tang: Network Adaptable Transmission Strategy Applied to H.264 : Digital Media and its Application in Museum and Heritages, 2nd Workshop, 2007

[5] Panayides, A.; Pattichis, M.S.; Pattichis, C.S.; Schizas, C.N.; Spanias, A.; Kyriacou, E.: An overview of recent end-to-end wireless medical video telemedicine systems using $3 \mathrm{G}$ : EMBC Annual International Conference of the IEEE , 2010

[6] Panayides, A.; Pattichis, M.S.; Pattichis, C.S.; Loizou, C.C.; Pantziaris, M.; Pitsillides, A. : Atherosclerotic Plaque Ultrasound Video Encoding, Wireless Transmission, and Quality Assessment Using H.264 : IEEE Transactions, 2011

77] Peter Rysavy, Transition to 4G : 3GPP Broadband Evolution to IMT-Advanced (4G), 3G Americas Publishes Research Report on 3GPP Mobile Broadband Evolution, September 2010

[8] Zhangyu Guan; Lei Ding; Melodia, T.; Dongfeng Yuan : On the Effect of Cooperative Relaying on the Performance of Video Streaming Applications in Cognitive Radio Networks : Communications (ICC), 2011 IEEE International Conference, June 2011

[9] Lei Ding, Scott Pudlewski, Tommaso Melodia, Stella N. Batalama, John D. Matyjas, Michael J. Medley: Distributed Spectrum Sharing for Video Streaming in Cognitive Radio Ad Hoc Networks: ADHOCNETS, Canada 2009

[10] Mansour, H.; Huang, J.W.; Krishnamurthy, V.: Multi-user scalable video transmission control in cognitive radio networks as a Markovian dynamic game; 48th IEEE Conference 2009

[11] Hsien-Po Shiang; van der Schaar, M.: Dynamic channel selection for multi-user video streaming over cognitive radio networks : Image Processing, ICIP, 15th IEEE Intern. Conference, 2008 
[12] Dramane Ouattara, Francine Krief, Mohamed A. Chalouf, Omessaad Hamdi : Spectrum Sensing Improvement in Cognitive Radio Networks for Real-Time Patients Monitoring : Wireless Mobile Communication and Healthcare; Springer 2013, p179-188.

[13] Jang-Ping Sheu; In-Long Lao : Cooperative routing protocol in cognitive radio ad-hoc networks : Wireless Communications and Networking Conference (WCNC), IEEE, 2012

[14] Chehata, A.; Ajib, W.; Elbiaze, H. : An on-demand routing protocol for multi-hop multi-radio multi-channel cognitive radio networks : Wireless Days (WD), 2011 IFIP, 2011

[15] Mishra, V.; Lau Chiew Tong; Chan, S.; Mathew, J., : MAC Protocol for Two Level QoS Support in Cognitive Radio Network: Electronic System Design (ISED), 2011 Intern. Symposium 2011.

[16] Mahmoud, H.; Yucek, T.; Arslan, H. : OFDM for cognitive radio: merits and challenges: Wireless Communications, IEEE, 2009

[17] Zhang Ju; Wan Senlin; A review of explicit model predictive control; Control Conference (CCC), 2012 31st Chinese, July 2012

[18] Jian-suo zhou; Zhi-Yuan Liu; Run Pei; A new nonlinear model predictive control scheme for discrete-time system based on sliding mode control; American Control Conference, 2001 